\title{
Analyse der Verformungen von Gewölbemauern
}

\begin{abstract}
Zusammenfassung: Die Berechnung von "normalen“ Sperrenverformungen unter den jeweils vorherrschenden Bedingungen und deren Vergleich mit der gemessenen Verformung ist ein wesentlicher Teil der Sperrenüberwachung. In vielen Fällen wird die Berechnung und der Vergleich automatisch durchgeführt und im Fall von zu großen Abweichungen ein Alarm ausgelöst. Der Hauptanteil an der Verformung von Bogensperren kommt von der Wasserlast und den Temperaturänderungen in der Sperre. Im Allgemeinen enthält die Verformung einen momentanen elastischen Anteil, einen zeitabhängigen reversiblen Anteil und einen irreversiblen Anteil. Grundsätzlich stehen für die Berechnung der Sperrenverformung deterministische Modelle, statistische Modelle oder hybride Formen aus den beiden zur Verfügung. Deterministische Modelle beruhen auf statischen Analysen, statistische Modelle auf Analysen der Verformungsmessungen.

Prediction of dam deformations can be based on deterministic models, purely statistical models or so called hybrid models which are a combination of the former two. Deterministic models rely on static analyses, statistical models on the statistical analysis of previous deformation data. For annual reservoirs, both water loading and temperature vary in cycles over a period of one year and it is extremely difficult to identify the various factors contributing to an observed deformation. This is particularly true for time- dependent reversible displacements due to the water loading and the portion caused by temperature variation.

The present article deals with the analysis of arch dam deformations based on hybrid models. Particular attention is given to the analysis of the deformations due to temperature fluctuation and to the long-term deformations. The proposed procedure is employed for the analysis of the deformations of the $186 \mathrm{~m}$ high Zillergruendl arch dam.
\end{abstract} Bei Jahresspeichern variieren sowohl der Stauspiegel als auch die Temperatur im Jahresrhythmus und es ist schwer, die beiden Anteile aus der resultierenden Verformung herauszufiltern.

Im vorliegenden Artikel wird ein hybrides Modell zur Analyse der Sperrenverformung eingesetzt. Besonders der Anteil der Temperaturverformung und die Langzeitverformung werden hervorgehoben. Das vorgestellte Verfahren wird bei der Analyse der $186 \mathrm{~m}$ hohen Zillergründlsperre eingesetzt und einige Ergebnisse dargestellt.

\section{Deformation analysis of arch dams}

Summary: Predicting "regular" dam deformations for the prevailing conditions and comparing them with observed deformations is an essential part of dam surveillance. In many cases prediction and comparison is carried out automatically and an alarm is triggered in the case of larger deviations.

The main contributors to the deformations of arch dams are water loading and the fluctuation of concrete temperature. In general, the deformations exhibit an instantaneous elastic part, a time dependent reversible part and an irreversible part.

\section{Einleitung}

Obwohl in einigen früheren Analysen von Jahresspeichern eine gute Übereinstimmung von gemessener Verformung und gerechneter Verformung erzielt wird, wird eine Frage nur vage beantwortet. Wie groß ist der Anteil der Wasserlast und wie groß ist der Anteil der Temperaturverformung an der Gesamtverformung? Der Grund für diese Schwierigkeit liegt darin, dass der zeitliche Verlauf der gemessenen Verformung und der Anteil aus der Wasserlast und der Anteil aus der Temperaturlast über das Jahr gesehen jeweils einen sinusförmigen Verlauf bilden. Bei der gemessenen Verformung sind Amplitude und Phase dieses Jahresganges direkt ersichtlich, nicht aber bei Verformung aus der Wasserlast, da diese auch einen verzögerten Anteil enthält (Kriechen) und bei der Temperaturverformung. Was die Temperaturverformung betrifft wird in einfachen statistischen Analysemodellen oft ein sinusförmiger Verlauf mit freier Amplitude und Phasenlage angesetzt („seasonal model") oder der Temperaturverlauf eines einzigen Thermometers verwendet (Lufttemperatur, Betontemperatur). Auf Grund der nicht vorgegebenen oder der ungeeignet vorgegebenen Phase ist keines dieser Modelle in der Lage, die Aufteilung zwischen Temperaturverformung und Verformung aus der Wasserlast korrekt vorzunehmen.

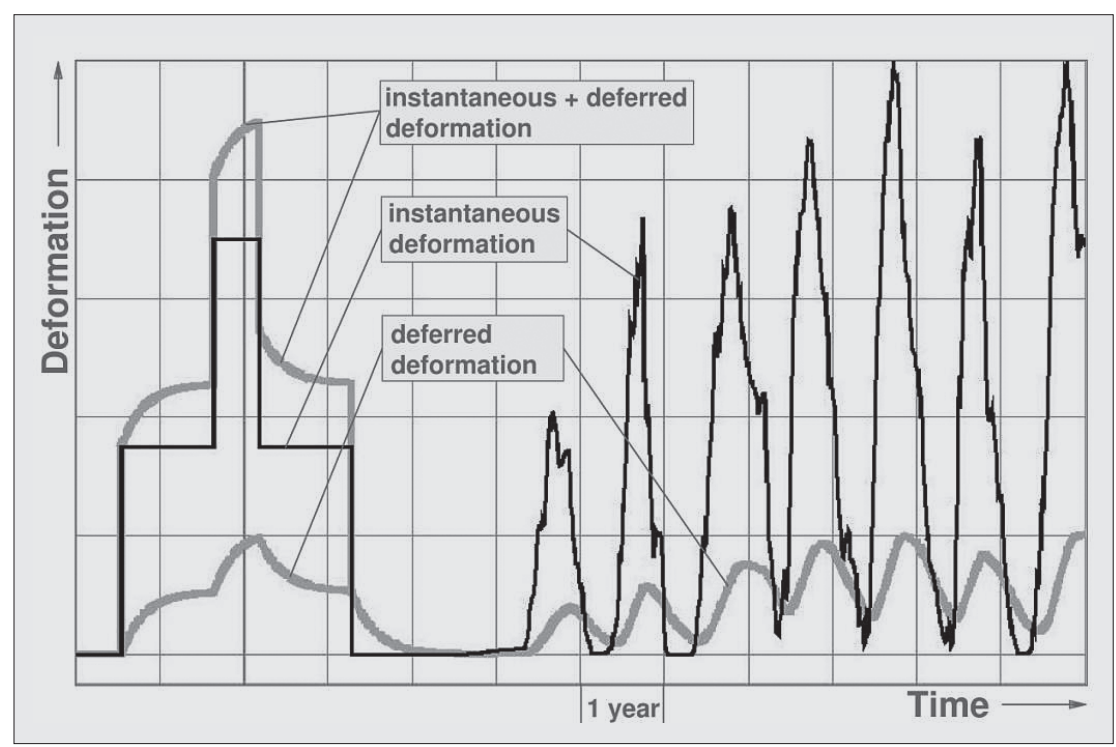

Abb. 1: Verzögerte Verschiebung für eine Stufenlast und eine typische Wasserlast im Jahresrhythmus. 
Das beschriebene Problem kann ge mindert werden, wenn die Verformungsanteile aus Temperatur und Wasserlast physikalisch sinnvoll beschrieben werden. Der momentane Anteil der Wasserlast wird mit Hilfe eines FE-Modells, der verzögerte Anteil mit einer Theorie für das Kriechen erfasst.

Besondere Anstrengung wird in die Erstellung eines guten Modells zur Temperaturverformung gelegt. Ausgehend von Betontemperaturmessungen in Oberflächennähe, werden die Wärmeleitungsgesetze benutzt, um die resultierende Verformung zu erhalten.

\section{Analytische Prozedur}

Um die Verformung von Bogensperren zu modellieren, ist es notwendig den funktionalen Zusammenhang zwischen der Verformung und den Variablen mit maßgeblichem Einfluss zu erstellen. In dieser Analyse werden nur die Variablen

- Stauhöhe $h$

- Betontemperatur $T$

- Zeit $t$

als maßgebend für die Verformung angesehen. Die erhaltenen Verformungen können eingeordnet werden in (Swiss Committee on Dams, 2003):

- momentan: nur die momentane Stauhöhe $h$ hat Einfluss auf die Verformung

- verzögert: die Geschichte der Stauhöhe $h$ hat Einfluss auf die Verformung

- reversible: die Verformung verschwindet (sofort oder später) nachdem die Last entfernt wird

- irreversible: die Verformung verschwindet nicht nach dem Entfernen der Last.

Die Temperaturverformung wirkt sofort in Bezug auf das Temperaturfeld in der Sperre (nur momentane Werte gehen in die Berechnung ein), aber zeitlich verschoben in Bezug auf einzelne gemessene Temperaturstellen (Geschichte der Temperaturmesswerte geht in die Berechnung ein).

Allgemein werden zwei grundsätzliche Methoden (und Mischformen davon) benutzt, um die Verformung durch die beeinflussenden Variablen zu beschreiben (Swiss Committee on Dams, 2003).

- die deterministische Methode, baut auf physikalischen Zusammenhängen auf. Die Anwendung dieser Methode auf die Verformung von Bogensperren geschieht typischerweise durch die
Diskretisierung von Geometrie, Materialgesetzen und Lasten durch Finite Elemente Methoden. Der springende Punkt dabei ist, dass keine Verformungsmessungen notwendig sind um die Methode anzuwenden

- die statistische Methode, approximiert die Messungen (in unserem Fall Verschiebungen) durch experimentelle Beziehungen mit mehr oder weniger physikalischer Bedeutung. Diese Methode erfordert Messungen über einen längeren Zeitraum und mit entsprechender Qualität (Datendichte).

In dieser Analyse wird eine Mischform der deterministischen und der statistischen Methode angewendet. Die Einflüsse der hydrostatischen Last und der Temperaturlast werden unter Verwendung der wesentlichen physikalischen Gesetze modelliert mit den in der Strukturanalyse üblichen Annahmen. In einem weiteren Schritt werden die modellierten Einflussfunktionen angepasst, um optimal zu den gemessenen Verschiebungen zu passen.

Die Anpassung geschieht am einfachsten mit Hilfe der bekannten statistischen Methode der multiplen linearen Regression (MLR). Sind die Beziehungen für die einzelnen Einflüsse (Basisfunktionen) einmal erstellt, wird die "beste“ lineare Kombination der einzelnen Einflüsse von der MLR gefunden. Das Attribut „beste“ besagt, dass die Summe der Quadrate der Differenzen zwischen der modellierten Verformung und der gemessenen Verformung (Residuen) minimiert werden. Mit anderen Worten, alle anderen linearen Kombinationen ergeben größere Residuen.

Es kann auch leicht gezeigt werden, dass die Residuen kleiner werden, wenn man zusätzliche Basisfunktionen in der MLR verwendet, auch wenn diese physikalisch keinen Sinn haben. Aber diese zusätzlichen Basisfunktionen bringen keine Verbesserung außerhalb des angepassten Bereiches. Die Analyse verkommt damit zu einer Blackbox, d.h. die Interpretierbarkeit der Ergebnisse wird schwieriger.

Der Vorteil der hybriden Methode ist, dass gute Ergebnisse mit einer kleinen Anzahl von Parametern erhalten werden können, da nur physikalisch sinnvolle Basisfunktionen verwendet werden. Die Verwendung von ausschließlich physikalisch relevanten Funktionen lässt uns auch erwarten, dass die Aufteilung der Verformung in einen hydrostatischen Anteil und einen thermischen Anteil besser wird (obwohl eine gewisse Unschärfe immer noch nicht zu vermeiden ist).

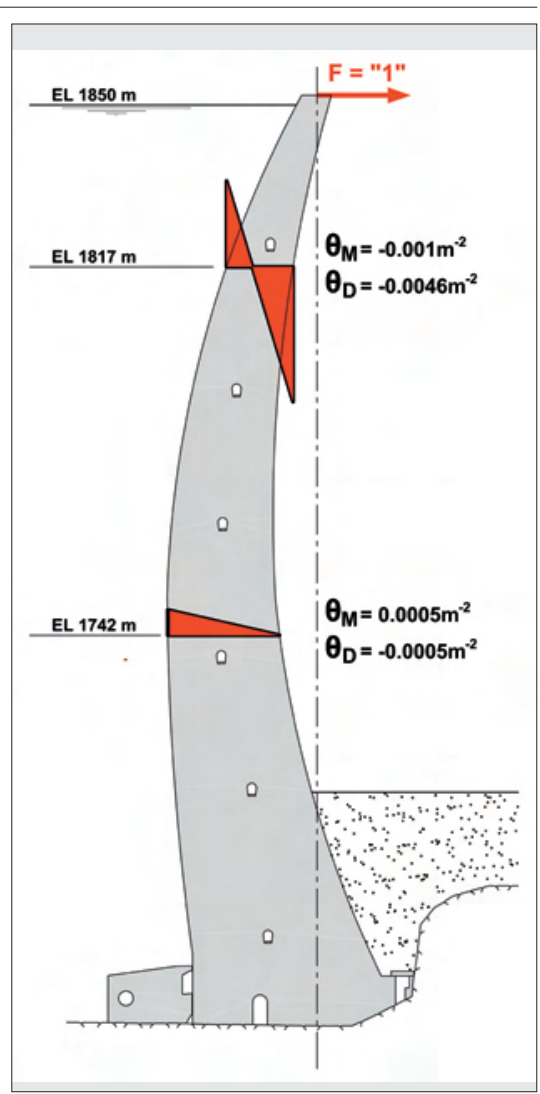

Abb. 2: Einheitskraft und resultierende Spannungsinvariante.

\section{Allgemeine Formulierung}

In der vorliegenden Analyse werden nur die von zwei Loten in radialer Richtung gemessenen Verschiebungen mittels einer multiplen linearen Regression analysiert. Ein Lot (das Sperrenlot) misst die relative Bewegung zwischen der Sperrenkrone und der Aufstandsfläche im Mittelblock. Das andere Lot (Felslot) misst die relative Bewegung zwischen der Aufstandsfläche und einem Punkt 80 Meter tiefer im Fels. Die grundlegende Formulierung für die vom Sperrenlot gemessene $\delta(t)$ Verschiebung lautet:

$\delta(t)=\delta_{h y}(t)+\delta_{c r}(t)+\delta_{t e}(t)+\delta_{l t}(t)+\delta_{c o}$

In dieser Formulierung ist $\delta_{h y}(t)$ die reversible momentane hydrostatische Verformung als Funktion der Zeit $t . \delta_{h y}(t)$ wird nur von der aktuellen (momentanen) Stauhöhe beeinflusst und nicht von der Geschichte der Stauhöhe.

Die reversible, verzögerte hydrostatische Verformung $\delta_{c r}(t)$ wird vom Kriechverhalten des Betons und des umgebenden Felsens als Reaktion auf eine länger einwirkende Last verursacht, d.h. die Geschichte des Stauspiegels $h(t)$ wird bei der 


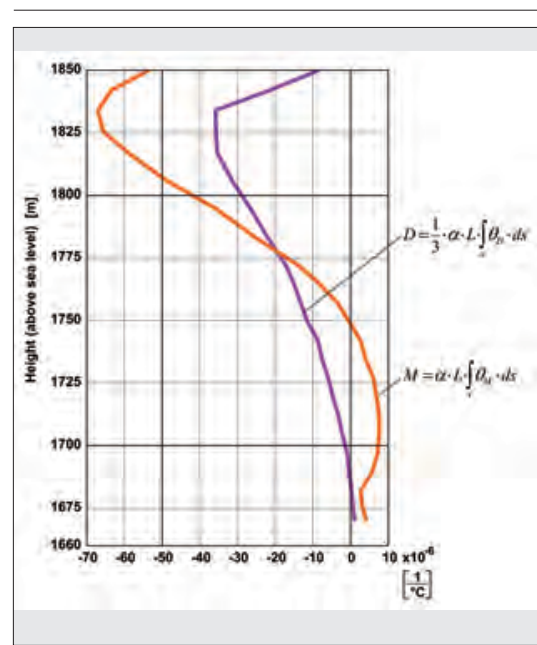

Abb. 3: Einfluss der mittleren Temperatur $T_{M}$ und des Temperaturgradienten $T_{D}$ auf die Kronenverschiebung.

Formulierung berücksichtigt (und nicht nur der momentane Stauspiegel).

Die reversible, von der Temperatur verursachte Verformung wird mit $\delta_{t e}(t)$ bezeichnet. Diese Verformung ist momentan in Bezug auf die Betontemperatur in der Sperre, die Temperatur im Fels wird vernachlässigt. Die Betontemperatur in der Sperre ist in Bezug auf die Oberflächentemperatur verzögert, das ist auch der Grund, warum die Temperaturverformung in Bezug auf die Oberflächentemperatur verzögert ist. Daher muss auch die Geschichte der Oberflächentemperatur berücksichtigt werden, um die entsprechende Verformung zu erhalten.

Alle irreversiblen Anteile an der Verformung (irreversibler hydrostatischer Einfluss, irreversibler Temperatureinfluss, etc.) werden durch den Term $\delta_{l t}(t)$ ausgedrückt, der hier Langzeiteinfluss genannt wird.

Schließlich wird die Konstante $\delta_{c o}$ eingeführt um z. B. den Nullpunkt der Messskala und allfällige weitere Konstanten zu berücksichtigen. Beim Lot im Fels wird der Temperaturterm nicht berücksichtigt, somit erhält man

$\delta(t)=\delta_{h y}(t)+\delta_{c r}(t)+\delta_{l t}(t)+\delta_{c o}$

(Anmerkung: In Gl. (1) und Gl. (2) werden die gleichen Ausdrücke für beide Lote verwendet, auch wenn sich ihre numerischen Werte unterscheiden.)

Um Gl. (1) und Gl. (2) in einer multiplen linearen Regression zu verwenden, müssen die einzelnen Terme zu

$\delta(t)=p_{h y} f_{h y}(t)+p_{c r} f_{c r}(t)+p_{t e} f_{t e}(t)+p_{l t} f_{l t}(t)+p_{c o} f_{c o}$

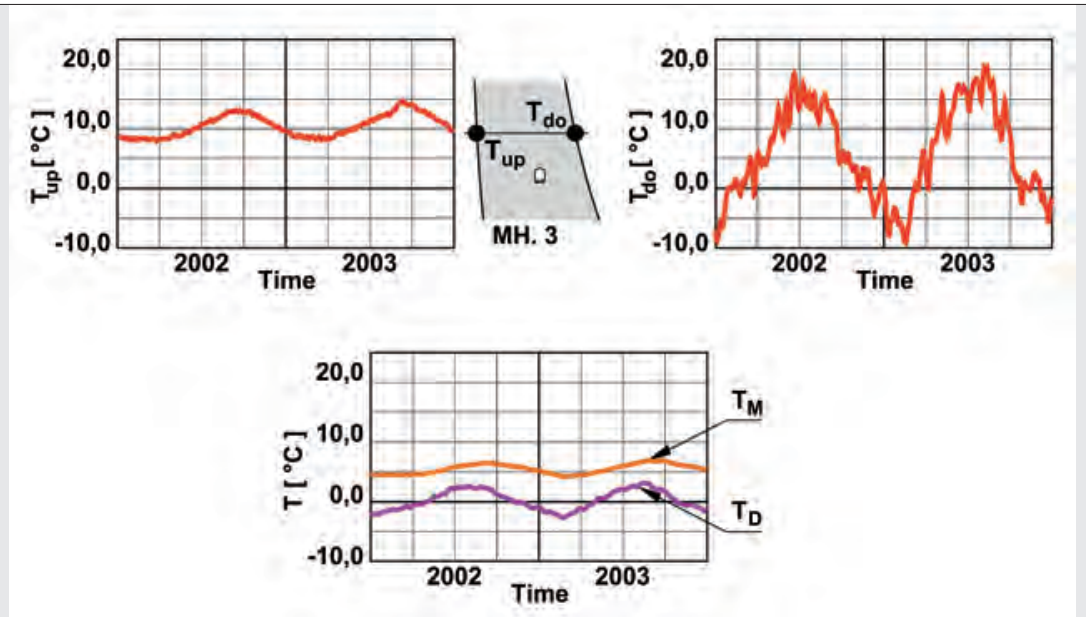

Abb. 4: Oberflächentemperaturen , mittlere Temperatur und Temperaturgradient an einem ausgewählten Querschnitt.

erweitert werden. In diesem Ausdruck sind $p_{h y^{\prime}} p_{c r}, \ldots, p_{c o}$ die sogenannten Regressionsparameter. Die Regressionsparameter sind Skalare, die beim Prozess der MLR bestimmt werden, so dass die Norm $\left[\delta(t)_{\text {measured }}-\delta(t)\right]=$ Minimum (Funktionen können als Verallgemeinerung von Vektoren gesehen werden, die Norm einer Funktionen wird berechnet wie die Norm (Betrag) eines Vektors).

Die Funktionen $f$.. $(t)$ werden in diesem Bericht Basisfunktionen genannt. Wenn sie in Gl. (3) nach sinnvollen physikalischen Gesetzen aufgebaut werden, erhält man die sogenannte hybride Formulierung des Problems, die die Physik mit der Statistik kombiniert.

\section{Verformung verursacht durch die hydrostatische Last}

\subsection{Momentane Verformung:}

Der momentane reversible Anteil $\delta_{h y}\left(t_{0}\right)$ zu einer bestimmten Zeit $t_{0}$ wird nur durch den Wert $h\left(t_{0}\right)$ bestimmt.

Die Beziehung zwischen der Verformung $\delta_{h y}$ und dem Stauspiegel $h$ wird durch ein 3d-Finite Elemente Modell durch die Berechnung der Verschiebungen für ausgewählte Werte von $h$ gewonnen. Die FE Ergebnisse werden durch eine Interpolation ergänzt. Für den Fall des Sperrenlots wird das Polynom

$\delta_{h y}=p_{h y} f_{h y}=p_{h y}\left(0,229 h-0,91 h^{2}+1,982 h^{3}-\right.$ $\left.1,148 h^{4}+0,782 h^{7}\right)$

gewählt. Für das Felslot ergibt sich aus der FE Approximation etwas einfacher $\delta_{h y}=p_{h y} h$

In beiden Gleichungen läuft $h=0$ bei leerem Speicher bis $h=1$ bei Stauziel.

Gl. (4) und Gl. (5) beschreiben die Form der Kurve, die Größe der Kurve wird durch die MLR bestimmt. In dieser Formulierung nimmt der Parameter $p_{h y}=105$ $\mathrm{mm}$ für die Sperre und $p_{h y}=3,5 \mathrm{~mm}$ für das Lot im Fels an. Diese Werte variieren leicht, abhängig vom Bereich der verwendeten Daten und der weiteren verwendeten Basisfunktionen in der MLR. Da die Basisfunktionen normiert sind $\left(f_{h y}=1\right.$ bei vollem Speicher $h=1$ ) können die Werte $p_{h y}=105$ und $p_{h y}=3,5$ als Verformung an der Krone und an der Aufstandsfläche interpretiert werden, die durch die hydrostatische Last bei Vollstau verursacht werden.

\subsection{Verzögerte Verformung (Kriechen)}

Der verzögerte reversible Anteil $\delta_{c r}(h)$ wird von der Stauspiegelgeschichte $h(t)$ beeinflusst (Lombardi et al., 2008). Die Ursache für die verzögerte Verformung ist das Kriechen in der Sperre und im Fels. Die klassische Formulierung die das Kriechen für eine Einheitslast beschreibt die zum Zeitpunkt $t=0$ startet, lautet

$\varphi(t)=\varphi_{\infty}\left(1-\mathrm{e}^{n t}\right)$

und beruht auf der Annahme eines viskoelastischen Materials. Der Materialparameter $\varphi_{\infty}$ wird durch die hybride Formulierung in der MLR bestimmt und $\eta=0.01$ wird auf der Basis von Laboruntersuchungen des Betons gewählt. Es ist nicht möglich $\eta$ in der MLR zu bestimmen, dazu 
wäre eine nichtlineare Regressionsrechnung notwendig.

Die Anwendung von Gl. (6) auf eine gegebene Lastgeschichte $F(\tau)$, oder in unserem Fall direkt auf die momentane Verschiebung $\delta(\tau)$ die mit $\tau=0$ startet gibt die Kriechverformung

$\delta_{e r}(t)=\int_{\tau=0}^{\tau=t} \varphi(t-\tau) \frac{\partial \delta(t)}{\partial \tau} \mathrm{d} \tau$

Nach partieller Integration der Gl. (7) und Einsetzen der Geschichte der momentanen Verformung aus der Wasserlast $\delta_{h y}(t)$ gibt die Formel

$\delta_{c r}(t)=-\int_{\tau=0}^{\tau=t} \varphi^{\prime}(\mathrm{t}-\tau) \delta_{h y}(\tau) \mathrm{d} \tau=$

$\eta \varphi_{\infty} \int_{\tau=0}^{\tau=t} e^{-\eta(t-\tau)} \delta_{h y}(\tau) \mathrm{d} \tau$

Gl. (8) ist viel benutzerfreundlicher als Gl. (7), da es jetzt nicht mehr notwendig ist die Last nach $\tau$ abzuleiten. Der linke Teil von Abb. 1 zeigt die verzögerte Verschiebung, wenn Gl. (8) auf eine stufenförmige Last angewendet wird (dargestellt für Anschauungszwecke), der rechte Teil der Abb. 1 zeigt das Ergebnis bei Anwendung auf die typische momentane Kronenverschiebung eines Jahresspeichers. Genauigkeitsuntersuchungen ergeben, dass die Geschichte der letzten zwei Jahre berücksichtigt werden soll.

\subsection{Verformung verursacht durch Temperaturänderung}

Die Verschiebung an einem Punkt in einer beliebigen Richtung verursacht durch die Temperaturvariation $T(t)$ kann durch

$\delta(t)=\alpha \int_{V} \Theta T(t) \mathrm{d} V$

dargestellt werden (Timoshenko et al., 1970). Das Integral erstreckt sich über den gesamten Sperrenkörper, die Temperaturänderung im Fels wird nicht berücksichtigt. $\alpha$ ist der lineare Temperaturausdehnungskoeffizient, $T$ ist das Temperaturfeld und

$\Theta=\sigma_{x x}+\sigma_{y y}+\sigma_{z z}$

ist das Feld der ersten Invarianten des Spannungstensors für eine Einheitskraft angesetzt an dem Punkt und in der Richtung der gesuchten Verschiebung.

Es stellt sich heraus, dass die Spannungen (und daher auch $\Theta$ ), die von der Einheitskraft $F=1$ verursacht werden, mehr oder weniger linear über die Dicke $L$ verlaufen. Das bedeutet $\Theta(x, y, z)$ kann durch

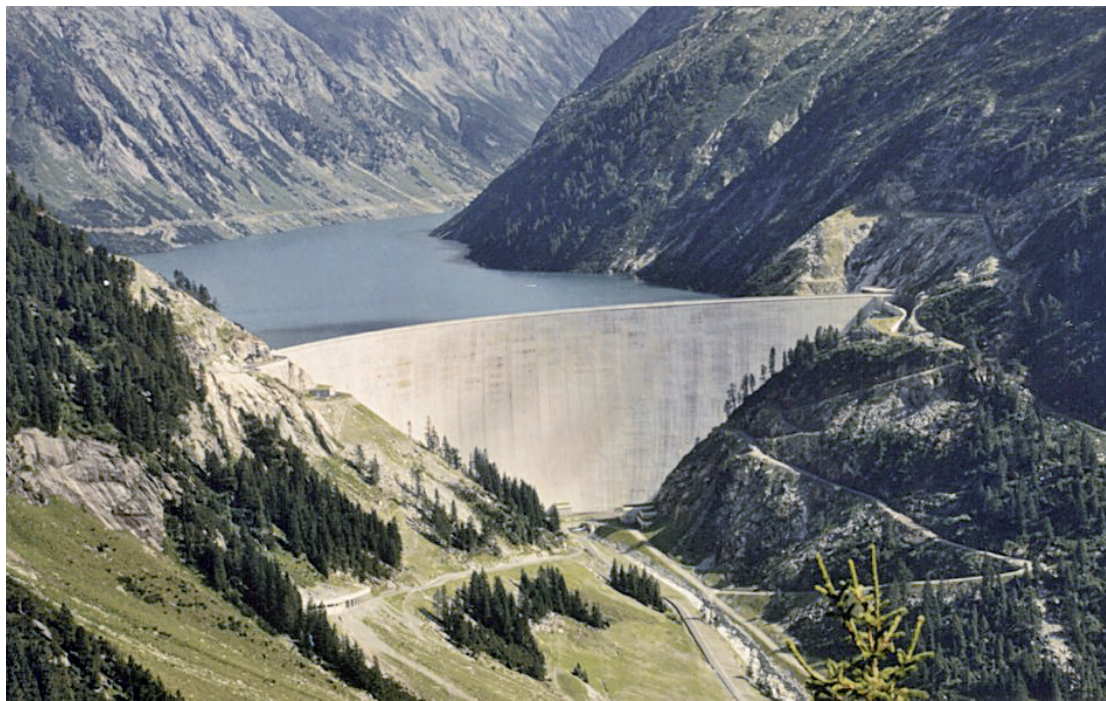

Abb. 5: Ansicht der Zillergründlsperre in Österreich.

seinen Mittelwert über die Dicke $\Theta_{M}$ und durch seinen Gradient $\Theta_{D}$ beschrieben werden (Abb. 2).

Wenn, entsprechend unserer Annahme, $\Theta$ linear entlang der Dicke $L$ verläuft, genügt es, den Temperaturverlauf durch den Mittelwert der Temperatur

$T_{M}(t)=\frac{1}{L} \int_{0}^{L} T(x, t) \mathrm{d} x$

und den Gradienten der Temperatur

$\mathrm{T}_{D}(t)=\frac{6}{L^{2}} \int_{0}^{L}\left(\mathrm{x}-\frac{L}{2}\right) T(x, t) \mathrm{d} x$

auszudrücken, auch wenn die Temperatur nicht linear entlang der Dicke variiert (Obernhuber et al., 2005). Mit diesen Annahmen gibt die Integration von Gl. (9) in radialer Richtung

$\int_{0}^{L} \Theta(x) T(x, t) \mathrm{d} x=L \Theta_{M} T_{M}(t)+\frac{L}{3} \Theta_{D} T_{D}(t)$

Wenn man weiter annimmt, dass die Temperaturen $T_{M}$ und $T_{D}$ entlang der Horizontalbögen konstant sind, kann Gl. (13) in tangentialer Richtung unabhängig von $T_{M}$ und $T_{D}$ unter Zuhilfenahme der numerischen Werte für $\Theta_{M}$ und $\Theta_{D}$ aus der FERechnung integriert werden

Die numerische Integration von $\Theta_{M}$ und $\Theta_{D}$ entlang der Horizontalbögen (der Sperre Zillergründl) ergibt die zwei Kurven $M$ und $D$ in Abb. 3, welche einen informativen Einblick auf die Reaktion der Sperre auf Temperaturänderung geben. Aus der Kurve $M$ kann man z. B. ablesen, dass eine Erhöhung von $T_{M}$ im Bogen mit der Höhe ca. $1830 \mathrm{~m}$ eine relativ große Verschiebung der Krone in Richtung Stau- raum bewirkt, eine Erhöhung von $T_{M}$ in der Höhe von ca. $1750 \mathrm{~m}$ bewirkt keine Kronenverschiebung.

Der letzte Schritt ist die Integration entlang der Sperrenhöhe. Unter Verwendung von $M$ und $D$ wird die Integration sehr einfach:

$\delta(t)=\int_{\text {Hegegt }} M(h) T_{M}(h, t) \mathrm{d} h+\int_{\text {Hegegt }} D(h) T_{D}(h, t) \mathrm{d} h$

Um Gl. (14) auszuwerten, werden $T_{M}$ und $T_{D}$ über die gesamte Sperrenhöhe gebraucht. Falls die Geschichte der Oberflächentemperatur an der Wasserseite $T_{u p}(t)$ und der Oberflächentemperatur an der Luftseite $T_{d o}(t)$ bekannt ist, können $T_{M}$ und $T_{D}$ durch jeweils ein Faltungsintegral bestimmt werden (Carslaw et al., 1959; Weber, 2005). In diesem Sinn kann die Verformung als momentan in Bezug auf das Temperaturfeld (oder auf $T_{M}$ und $T_{D}$ ) bezeichnet werden, aber als verzögert in Bezug auf die Oberflächentemperatur $\left(T_{u p}(t)\right.$, $T_{d o}(t)$ ).

Genauigkeitsuntersuchungen ergeben, dass die Geschichte der Oberflächentemperaturen für die Zillergründlsperre etwa zwei Jahre in die Vergangenheit berücksichtigt werden sollen. Dieser Zeitbereich hängt hauptsächlich von der Dicke der Sperre $a b$.

Ein Problem taucht auf, falls die Temperatur nicht an der Oberfläche, sondern tiefer in der Sperre gemessen wird. In diesem Fall hat man es mit einem inversen Problem der Wärmeleitung zu tun, das besondere Maßnahmen verlangt, z.B. eine Glättung der Messdaten (Weber, 2005). Einfach die Lufttemperatur an Stelle der 


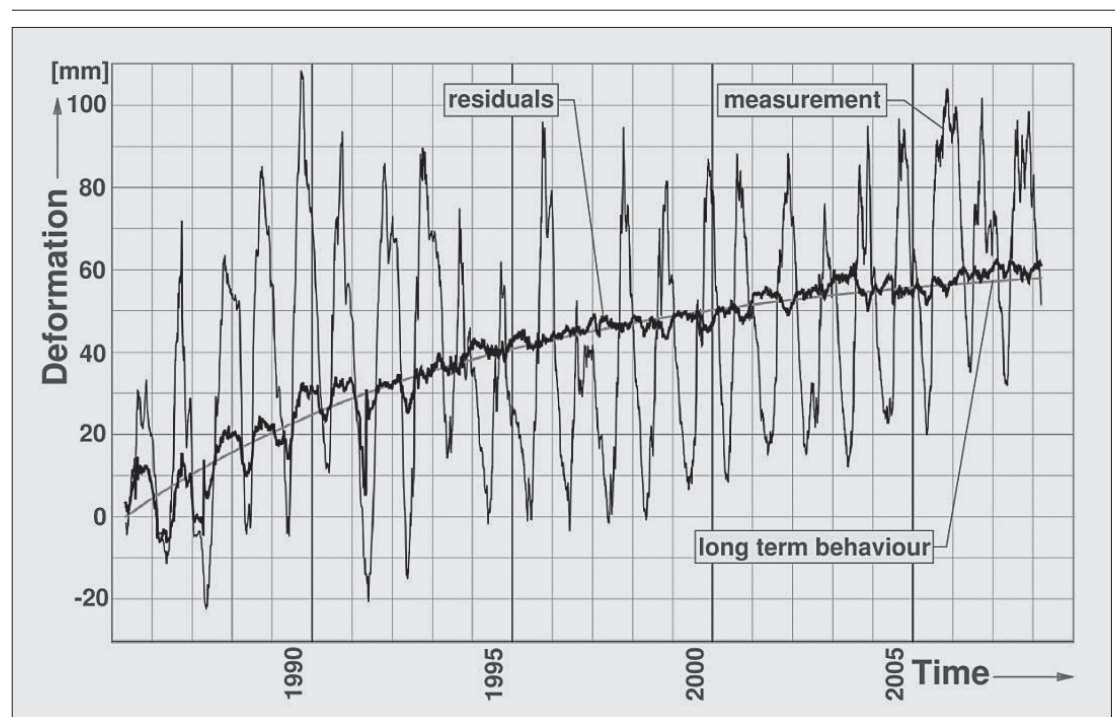

Abb. 6: Langzeitverhalten der Sperre, Messungen, Residuen.

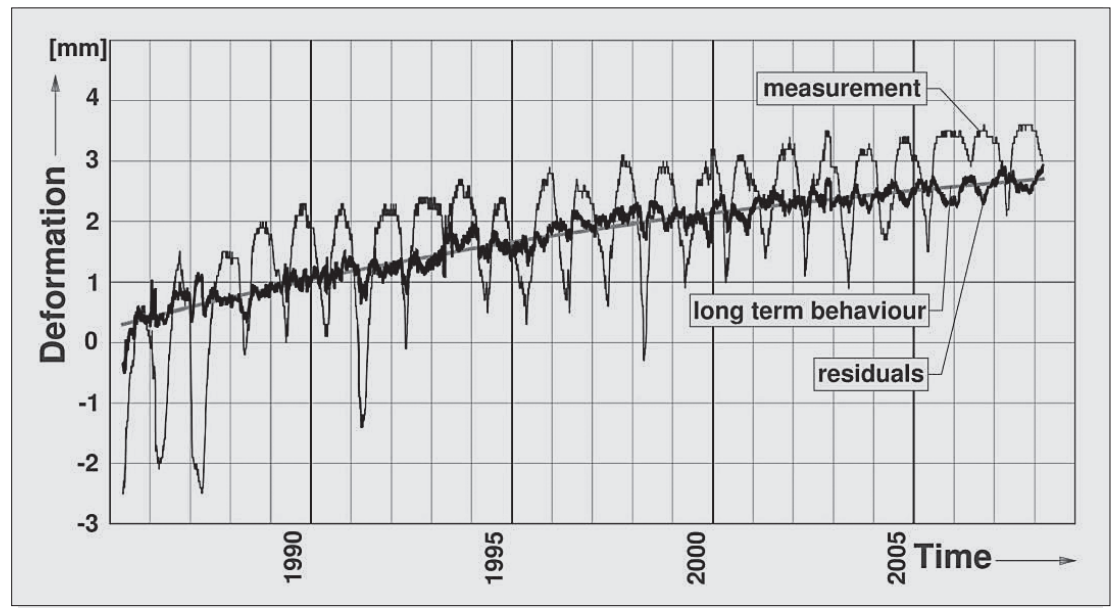

Abb. 7: Langzeitverhalten des Felsens, Messungen, Residuen.

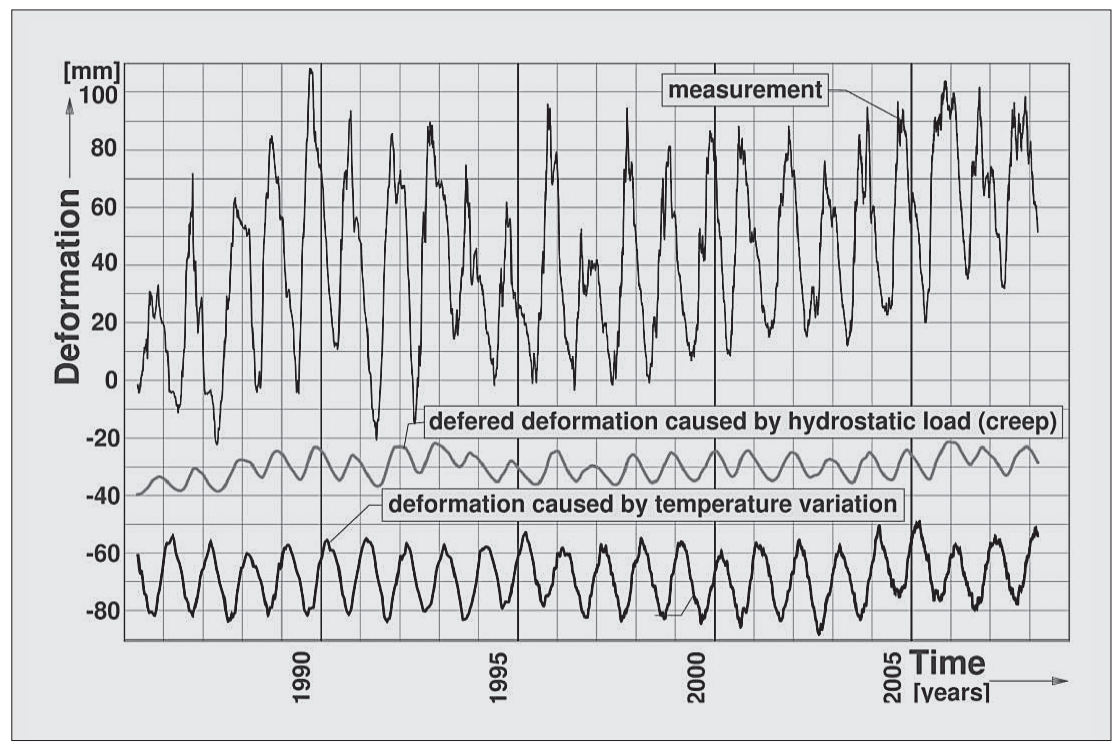

Abb. 8: Messung, Kriechen und Temperaturbewegung.
Oberflächentemperatur zu verwenden kann zu Fehlern führen, da die Oberflächentemperatur und die Lufttemperatur weit auseinanderklaffen können.

In unserem Fall errechnen wir die Oberflächentemperaturen von Thermometern in einer Tiefe von ca. $0,3 \mathrm{~m}$ in einem Messhorizont und in einer Tiefe von ca. 1,5 $\mathrm{m}$ in einem anderen Horizont durch Verwendung der gemessenen Temperaturgeschichte über zwei Monate. In den Horizonten zwischen den Messhorizonten wird die Oberflächentemperatur interpoliert. Anschließend wird aus der Geschichte der Oberflächentemperaturen die mittlere Temperatur $T_{M}$ und der Temperaturgradient $T_{D}$ ermittelt, wie in Abb. 4 skizziert ist (Obernhuber et al., 2005).

\subsection{Irreversible Verformung}

Der irreversible Anteil $\delta_{l t}(t)$ von allen Lasten (Wasserlast, thermische Last, andere) wird mit als maßgeblicher Variable modelliert.

$\delta_{l t}(t)=p_{l t} f_{l t}(t)=p_{l t}\left(1-e^{-\kappa t}\right)$

Dieser Langzeitterm inkludiert alle Verformungen, die nicht von anderen Basisfunktionen aufgefangen werden. Unglücklicherweise gibt es eine Interaktion mit dem Kriechverhalten, was zu numerischen Unsicherheiten beim Kriechparameter $p_{c r}$ und beim Langzeitparameter $p_{l t}$ führt. Die Statistiker sprechen in diesem Zusammenhang von einer starken linearen $\mathrm{Ab}$ hängigkeit der beiden Basisfunktionen $f_{l t}$ und $f_{c r}$. Die Krümmung der Basisfunktion ist durch den Parameter $\kappa$ gegeben, der aber nicht in der MLR bestimmt wird, sondern in einer Vorstudie unter Verwendung einer nichtlinearen Regressionsrechnung mit $\kappa=1 / 3448$ Tage ermittelt wird, d. h. ca. $2 / 3$ der Langzeitbewegung werden in den ersten 10 Jahren erreicht.

\section{Fallstudie - Zillergründlsperre}

\subsection{Zillergründlsperre}

Die Zillergründl Sperre (siehe Abb. 5) ist eine $186 \mathrm{~m}$ hohe Bogenmauer mit einer Kronenlänge von 506 m (Austrian National Committee on Large Dams, 1991) und wurde von 1979 bis 1985 gebaut. Der Stauraum beträgt $89 \times 10^{6} \mathrm{~m}^{3}$ und wird als Jahresspeicher genutzt. Der Stauraum ist im Frühling mehr oder weniger leer, während der Stauspiegel im Herbst sein Maximum erreicht. Die Sperre ist auf massivem Gneis gegründet und hat einige 
interessante Besonderheiten, wie z. B. eine Basisfuge über ca. ein Drittel des Querschnittes an der Aufstandsfläche. Die Sperre ist mit einem umfassenden Monitoringsystem ausgerüstet, das u. a. Lote und Thermometer in verschiedenen Querschnitten enthält.

\subsection{Analyse der Verformungen}

In Abb. 6, 7 und 8 werden einige Ergebnisse aus der Anwendung der oben beschriebenen Methode auf die Zillergründlsperre dargestellt. Die Auswertung beruht auf täglichen Messwerten für die Lote in der Sperre und im Fels seit dem Jahr 1986, also kurz nach Fertigstellung der Sperre. Bereits die Plots der Messungen über bisher etwa 23 Jahre zeigen die Tendenz der Sperre zu einer langzeitlichen Bewegung zur Luftseite. Nach dem rechnerischen Entfernen des momentanen und des verzögerten Staueinflusses und des Temperatureinflusses ist die Langzeitbewegung der Sperre (Abb. 6) und des Untergrundes (Abb. 7) viel deutlicher erkennbar als in den Messungen (Residuen). Der modellierte Langzeittrend nach Gl. (15) ist als glatte Linie erkennbar.

Bis zum Jahr 1992 gab es in der Sperre einige Injektionsarbeiten. Diese Arbeiten führten auch zu Änderungen im Sperrenverhalten und könnten der Grund für die größeren Residuen bis zum Jahr 1992 sein, danach variieren die Residuen deutlich weniger.

Die irreversible Langzeitbewegung der Sperrenkrone gegenüber der Aufstands- fläche erreicht ca. $28 \mathrm{~mm}$ seit dem Ende der Injektionsarbeiten und die Bewegung der Aufstandsfläche ca. $2 \mathrm{~mm}$.

In Abb. 8 wird die gemessene Bewegung zusammen mit der verzögerten Bewegung aus der Wasserlast (Kriechen) und der Temperaturbewegung dargestellt. Die Temperaturbewegung wird aus der Geschichte von vier Thermometerablesungen in der Sperre nahe der Sperrenoberfläche berechnet. Der Temperaturausdehnungs-koeffizient, also der Maßstab der Temperaturverformung, wird in der MLR bestimmt. Im Verlauf der Temperaturbewegung kann man die Auswirkung des besonders warmen Sommers im Jahr 2003 und zwei kalte Winter in den Jahren 2005 und 2006 erkennen.

\section{Zusammenfassung}

Die vorliegende Analyse von Verformungsmessungen stützt sich auf eine hybride Methode, die die Ergebnisse der strukturalen Analyse mit statistischen Methoden kombiniert. Die strukturalen Ana- lysen werden eingesetzt, um die physikalischen Beziehungen zwischen Verformung und Wasserlast und zwischen Verformung und thermischer Last zu erstellen. Diese Beziehungen werden dann in einer statistischen Analyse (MLR) der gemessenen Verschiebungen als sogenannte Basisfunktionen eingesetzt.

Mit dieser Kombination erhält man gute Resultate bei einer kleinen Anzahl von Parametern in der MLR Analyse. Damit ist auch eine optimale Aufteilung der Verformung in die verschiedenen Einflüsse erreichbar: momentane Verformung durch die Wasserlast, verzögerte Verformung durch die Wasserlast, Einfluss der Temperatur auf die Verformung und Ermittlung von Langzeiteffekten.

\section{Korrespondenz:}

F. Perner, P. Obernhuber

Verbund-Austrian Hydro Power AG

Am Hof 6a

E-Mail: Franz.Perner@Verbund.at

\section{LITERATUR}

National Committee on Large Dams (1991) Dams in Austria

Lombardi G, Amberg F, Darbre GR (2008) Algorithm for the prediction of functional delays in the behaviour of concrete dams, Hydropower \& Dams, Issue 3

Obernhuber P, Perner F (2005) Displacements in concrete dams caused by temperature variation. Hydropower \& Dams, Special Issue-Hydro 2005, pp. 80-86

Swiss Committee on Dams (2003) Methods of Analysis for the Prediction and the Verification of Dam Behaviour. Eau-Energie-Air

Timoshenko SP, Goodier JN (1970) Theory of Elasticity, McGraw-Hill, USA

Weber B (2005) variation in concrete dams based on one-dimensional heat conduction, (not published) 